\title{
Development and validation of a questionnaire in parenting patterns : evidence from Tamil culture in Northern Sri Lanka
}

\author{
Saseela Subaskaran $^{1^{*}}$ and Aindralal Balasuriya ${ }^{2}$ \\ ${ }^{I}$ Ministry of Health, Sri Lanka. \\ ${ }^{2}$ Faculty of Allied Health Sciences, General Sir John Kotelawala Defence University, Rathmalana, Sri Lanka.
}

\begin{abstract}
Adolescent health issues such as substance abuse, high risk sexual behaviours and psychosocial problems are becoming a threat globally. The World Health Organization has identified parenting interventions as a strategy to improve adolescent health. This study aimed to develop and validate a tool to understand parenting practices for Northern Province Tamil culture in Sri Lanka. A Parenting Patterns Questionnaire (PPQ) with three subscales for three parenting dimensions; 'Connection', 'Control' and 'Respect', was constructed in accordance with parenting styles and parenting tools used in Western countries and by expert opinion. Item analysis was conducted to establish the internal consistency and exploratory factor analysis was conducted to check whether the items are assigned to the correct subscales. Construct validity of the PPQ was confirmed by demonstration of a moderate to good correlation with two hypothetical constructs, namely the wellbeing of the children demonstrated by the scores of Strength and Difficulties Questionnaire (SDQ), and educational performance of the children assessed by the marks of previous term examination. The internal consistency of the PPQ and subscales was satisfactory (Cronbach's alpha > $0.7)$. The test re-test reliability also was satisfactory for PPQ (Pearson's correlation Coefficient $>0.9$ for selected items). The correlations between the parenting quality assessed by the PPQ and the above mentioned constructs showed a moderate correlation $(r>0.3)$. This tool (PPQ) has the potential to be used by researchers to assess parenting in selected dimensions ('Connection', 'Control' and 'Respect') in Sri Lankan Tamil culture.
\end{abstract}

Keywords: Parenting questionnaires, adolescent

\section{INTRODUCTION}

Parenting is defined as rearing of a child or children, especially the care, love and guidance given by the parent (American Heritage Dictionary, 2009). It is a construct which cannot be defined easily and is understood by different cultures differently. Parenting is predominantly seen as a task about the socialisation of the children within the community that considers children in relation to their families, neighbourhood and the wider community (White, 2005).

The main dimensions of parenting identified by the World Health Organization (WHO, 2007) are Connection (love) which is an emotional bond between parent and child; Behaviour control which includes regulation, monitoring, structure and limit-setting, encompassing parents' actions aimed at shaping or restricting child's behaviours; Respect for individuality (respect) which involves allowing the child to develop a healthy sense of self, apart from his or her parents; Modelling for appropriate behavior (model) which includes modelling according to the social norms and culture; and Provision and Protection (provide) which refers to parents' provision of the resources that they can and seeking out resources when they cannot provide.

Baumrind (1967) presented a method of classification of parenting styles depending on responsiveness and demanding. She identified three parenting styles; Authoritative, Authoritarian and Permissive.

Maccoby and Martin (1983) expanded the above three into four parenting styles; Authoritative, Authoritarian, Permissive and Neglectful styles. The Authoritative parenting style is characterised by high parental responsiveness and high parental demands. In Authoritarian Parenting style, the parent is demanding but not responsive. The parent is responsive but not demanding in Permissive style. The parent is neither 
responsive nor demanding in the Neglectful style. Numerous studies show that the Authoritative style brings good outcomes among adolescents (Stafström, 2014; Hoffman and Bahr, 2014), while some other researchers have found out that in some cultures and ethnicities (Asian American families) Authoritarian style may be associated with more positive child outcomes (Santrock, 2014).

\section{LITERATURE REVIEW}

White (2005) discusses the constraints in assessing 'parenting'. Assessment of parenting or parenting capacity or competence takes place in the West for the best interest of the child, mainly in the context of courts and to take decisions of the provision of care for a child in future. Even though it is essential to do a parenting assessment, it is a difficult task to produce a correct and unbiased report for those purposes.

Parenting practices vary across cultures and societies (Sanders, 2005). Russell et al. (2010) summarised many studies done in white American families and Asian - American families and highlighted the differences between them. They have further pointed out that what is accepted by white Americans as good parenting is 'Authoritative parenting'. According to the studies, it was clear that even though in the Western culture Authoritarian parenting (less warmth and high control) is seen as likely to damage adolescent's wellbeing, it is not necessarily more beneficial than Authoritative parenting. It is proved that the first-generation Chinese youth from Authoritarian homes do just as well in school as those from Authoritative homes. Further, the book stressed on introducing new dimensions of parenting such as 'sacrifice' which is perceived by the children in Asian culture. Evidence provided by researches show that Chinese-American youth think of support and control differently than White or Filipino Americans. Chinese adolescents reported that the parents show their love by instrumental support (by provision of needs and sacrifice for children) than verbal for example "I love you". For them parental sacrifice is central for the support (Russell et al., 2010).

Among Sri Lankan Tamil families, family interests are prioritised over individual interests (Sivarajah, 1998; Kendall, 1989). The advice on child rearing is obtained from older relatives and friends. The Tamil family unit revolves around children who are objects of love and affection. Parents make decisions on behalf of their children who are raised into obedience (Kendall, 1989; Sivarajah, 1998).
In a study among Sri Lankan Tamils in Canada, the results revealed that parents have deep concern for their children's well-being and among parental concerns, education ranked the highest (Tyyskä and Colavecchia, 2001). Parents also expressed concern over children falling in with the wrong crowd and engaging in "deviant" activities. They are also concerned about the negative influence of Western materialistic and consumerist values that were perceived as a threat to traditional Tamil values such as respect for elders and family loyalty.

Generally, in Tamil society, boys are granted more freedom than girls. There was particular concern over the safety and good reputation of daughters. The parents treat their daughters as the "flag-bearer of their culture" who needed to be chaste, dress appropriately and participate in cultural customs (Tyyskä and Colavecchia, 2001).

Effective parenting capacity assessment is not only dependant on the development of appropriate definitions, tools and systems of data collection but also on knowledge, skills, organisational support and supervision and training of heath and social care staff (London Safeguarding Children Board, 2010).

Several validated tools are available in the Western culture to assess parenting. The tools were developed to assess parenting quality of parents at different ages of the children; some tools are parental report and others are children's report.

The Parenting Authority Questionnaire (PAQ) (Buri, 1991) is a tool that describes the parenting styles proposed by Baumrind (1967) and is used in many researches. It consists of 30 items per parent and yields Permissive, Authoritarian and Authoritative scores for both the mother and the father; each of these scores is derived from the phenomenological appraisals of the parents' authority by their son or daughter. Buri (1991) conducted seven studies to test the PAQ's reliability, internal consistency, content-related validity, criterion-related validity, discriminant-related validity, and its correlations with the Marlowe-Crowne Social Desirability Scale. The results of these studies showed the PAQ to have highly respectable measures of reliability and validity. The PAQ is useful for assessing the parental authority exercised by both mothers and fathers (Buri, 1991).

Arnold et al. (1993) developed a rating of scale to measure dysfunctional discipline practices in parents of children aged 18-48 months. They established the testretest reliability and internal consistency as reliability measures and construct validity. Three stable factors (laxness, over reactivity and verbosity) of dysfunctional 
discipline were identified. Among the 30 items, 17 were not under any factor. The scale exhibited adequate alpha values and test-retest correlations $(>0.9)$ for three subscales and for the overall scale. Construct validity was established by showing correlations in the expected directions with the child behaviour problems and marital discord. The child behaviour problems were assessed by Child Behaviour Check List (CBCL) (Achenbach and Edelbrock, 1983) and the marital discord was assessed using Short form of Marital Adjustment Test (SMAT) (Locke and Wallace, 1959). As expected, total scores and the scores for each factor were significantly related to levels of child misbehaviours reported by the mothers on CBCL. A significant negative relationship was obtained with SMAT scores.

Campis et al. (1986) constructed a scale to assess the parental locus of control called 'The Parental Locus of Control (PLOC) Scale'. Two studies were done for the development and validation of PLOC scale. In the first study, the scale was developed and administered to 147 parents of elementary school children. Data analysis yielded a 5 factor solution (parental efficacy, parental responsibility, child's control on parents' life, parental belief in fate or chance and parental control of child's behaviour) with acceptable indices of reliability. The second study was carried out to assess the construct and discriminant validity of PLOC scale. The construct validity was established against 7 scales which predicted a correlation with the sub scales of PLOC. Five out of eight predictions were confirmed. To assess the discriminant validity, comparison between 2 groups of parents was made on their responses to PLOC. Group A consisted of 60 parents who reported no difficulties in the parenting role and group B consisted of 45 parents who requested professional services for parenting problems or whose children were identified as having emotional or behavioural problems. As predicted, a multivariate analysis of variance indicated that the two groups differed significantly $(\mathrm{p}<0.002)$.

Sanders (2005) designed a study to examine the psychometric properties of the Parent Behaviour Questionnaire (PBQ) and the Parent Behaviour Frequency Questionnaire (PBFQ) which measure specific parenting behaviours and the frequency of those behaviours respectively. Several statistical analyses were used to assess the psychometric properties of the scales. Pearson correlation coefficients were utilised to assess the test re-test reliability as well as the correlations among the six subscales, that is, the six parent role characteristics (i.e., bonding, discipline, education, general welfare and protection, responsively and sensitivity) of the PBQ and the PBFQ. Results indicate that both instruments demonstrate adequate test re-test reliability and internal consistency.

A Parent Practices Scale (Strayhorn and Weidman, 1988) was developed to describe the patterns of the parenting of adolescents. The scale consisted of 36 items with a 7 point adjective scale based on situations or interactions that occur with parents and children. The items were on 3 dimensions; warmth, control and harsh parenting.

As described above, parenting has a big role in shaping the future. But it is very difficult to assess since there is no accepted upon best style of parenting. Furthermore, parenting is affected by the context and the culture too.

Therefore, this study was designed with the objective to develop a tool to describe parenting in Tamil culture in Northern Province of Sri Lanka.

\section{METHODOLOGY}

The study was carried out in Northern Province of Sri Lanka.

The objective of the study was to develop and validate a questionnaire to describe the patterns of parenting by parents of 10 year old school going adolescents in the Northern Province.

The study consisted of two stages:

1. Development of a scale to describe parenting, targeted to gather the information from 10 year old adolescents

2. Validation of the developed scale

The development of the scale, named Parenting Patterns Questionnaire (PPQ) which is a child version of parenting questionnaire, for 10 year old adolescents, was done in five stages.

Stage I: Devising a range of items suitable for the preliminary draft of the PPQ from literature and by generating new items by Focus Group Discussions (FGD) and Key Informant Interviews (KII).

Stage II: Formulation of items and response categories for the preliminary draft of the PPQ. 
Stage III: Pre-testing of the questionnaire.

Stage IV: Application of item reduction procedures (reliability analysis) to select the most suitable set of items for the final draft of the PPQ that will contribute to a good internal consistency.

Stage V: Exploratory factor analysis to confirm the items are assigned to the correct subscales.

All the stages in the instrument development and validation process were designed according to the guidelines proposed by Streiner and Norman (2003). The summary of the development and validation of PPQ is shown in Table 1.
The items adopted from the existing instruments were incorporated into list 1 (Tamil) and the items derived from FGDs and KIIs were incorporated into list 2. Items from both the lists 1 and 2 were looked for any duplication and the duplicated items were removed to form a final common list which ended up with 50 items. The draft was circulated among the same panel of experts who participated for the development of item list 2. Finally, the Delphi technique was used to develop the preliminary draft of the PPQ consisting of 35 items.

Subscales were developed based on three dimensions identified as important by the World Health Organization (WHO, 2007). Therefore, the items were assigned under three sub scales:

Table 1: The summary of steps of development and validation of PPQ

\begin{tabular}{|c|c|c|}
\hline Steps & Procedure of steps & Participants \\
\hline \multirow[t]{2}{*}{$\begin{array}{l}\text { Step I: } \\
\text { Devising the items }\end{array}$} & $\begin{array}{l}\text { Selection of suitable items from exist- } \\
\text { ing instruments by an opinion survey } \\
\text { among a panel of experts (item list 1) }\end{array}$ & $\begin{array}{l}\text { A consultant community physician, a } \\
\text { consultant psychiatrist, a psychologist, } \\
\text { a sociologist, an educationist and } 2 \\
\text { religious leaders }\end{array}$ \\
\hline & $\begin{array}{l}\text { Generation of new items using FGDs } \\
\text { and KIIswith relevant people (item } \\
\text { list 2) } \\
\text { Common item list (50 items) }\end{array}$ & $\begin{array}{l}\text { Adolescents, parents, school principal, } \\
\text { psychologist, counseling teacher, a } \\
\text { sociologist and a religious leader. }\end{array}$ \\
\hline \multirow[t]{3}{*}{$\begin{array}{l}\text { Step II: } \\
\text { Development of the preliminary draft } \\
\text { of PPQ }\end{array}$} & $\begin{array}{l}\text { Common item list ( } 50 \text { items) } \\
\text { (Expert panel \& establishment of judg- } \\
\text { mental validity) }\end{array}$ & $\begin{array}{l}\text { A consultant community physician, a } \\
\text { consultant psychiatrist, a psychologist, a } \\
\text { sociologist, an educationist }\end{array}$ \\
\hline & $\begin{array}{l}\text { Preliminary draft of the PPQ ( } 35 \text { items) } \\
\text { Formulation of subscales and response } \\
\text { categories and scoring }\end{array}$ & $\begin{array}{l}\text { Tamil speaking students from Colombo } \\
\text { district }\end{array}$ \\
\hline & Pre-testing & \\
\hline $\begin{array}{l}\text { Step III: } \\
\text { Development of the final draft of PPQ }\end{array}$ & $\begin{array}{l}\downarrow \\
\text { Final draft of PPQ (27 items) }\end{array}$ & $\begin{array}{l}\text { Sample from the } 10 \text { year old students } \\
(n=150) \text { from Kilinochchi district }\end{array}$ \\
\hline $\begin{array}{l}\text { Step III: } \\
\text { Validation of PPQ }\end{array}$ & Establishment of construct validity & $\begin{array}{l}\text { Same sample as item analysis and factor } \\
\text { analysis }\end{array}$ \\
\hline
\end{tabular}


- Subscale 1 included 19 items to describe 'Connection'.

- Subscale 2 included 9 items to describe 'Control'.

- Subscale 3 included 7 items to describe 'Respect'.

Each item in the questionnaire was worded as a close-ended question which was followed by a 5-point adjectival scale. The response choices for each individual item were designed to correspond with the item stem. Respondents were expected to indicate the extent or the frequency of occurrence of the condition or experience described by a particular item, by selecting the most appropriate response alternative that followed the item.

Three types of response alternatives designed for the scale were 'always or every day/ frequently/sometimes/ rarely/ never'; 'completely/ to a greater extent/ to some extent/ to a lesser extent/ not at all'; and 'very frequently/ frequently/ sometimes/ rarely/not at all', depending on the item stem.

Scoring and deciding the cut off marks was done with the help of existing instruments.

\section{INTERNAL CONSISTENCY, FACTOR STRUCTURE AND CONSTRUCT VALIDITY}

A sample of 150 ten year old students was recruited for this study, 75 boys and 75 girls, 50 students from each type of school (type AB, C and type II). They were given the PPQ (preliminary draft with 35 items) and Strength and Difficulties Questionnaire (SDQ) and were asked to complete them. Their previous term exam performance was extracted to a data sheet.

Item selection procedures were applied to :

- reduce the number of items to a manageable size.

- determine the appropriateness of the items in each subscale.

The preliminary draft of PPQ which consisted of 35 items was applied with item selection procedures and the items which reduced the internal consistency and homogeneity were removed. After the removal of the unsuitable items, the PPQ consisted of 31 items (subscale 1: 17 items; sub scale 2: 8 items; subscale 3: 6 items).

Factor analysis: Factor analysis was used by researchers to investigate the factor structure, for test validation by factoring the test with best known marker variables
(Kline, 1994) and to assess whether the items in a multidimensional scale are assigned to the right subscales, by means of their factor loadings (Streiner \& Norman, 2003).

The final draft of the PPQ was subjected to exploratory factor analysis to confirm its multidimensionality and to check whether the items are in the right scales measuring the different dimensions of caregiver burden.

Kaiser-Meyer-Olkin (KMO) test and Bartlett's Test of Sphericity were used to determine the appropriateness of factor analysis.

The KMO test measures the sampling adequacy while the Bartlett's Test of Sphericity tests the null hypothesis that the original correlation matrix is an identity matrix. Factor analysis is appropriate when the Bartlett's test shows a significant result.

The Principal Component Analysis (PCA) was performed to identify the number of factors to be rotated. The number of factors arose from the PCA were decided based on the eigenvalues and Scree test. In addition, re-formulation of subscales also was done according to the PCA. However, an attempt was made to retain the original categorisation as far as possible. (This categorisation was thought to be useful in subsequent designing of interventions for parents.) Six items were removed from the 31 item scale derived after the item selection procedures and the final PPQ ended up with 27 items.

Test-retest reliability: The test - retest reliability of the instrument was measured by re-administering the instrument after two weeks from the initial administration to a group of 30 children who were selected for the validation part of the study.

\section{VALIDATION OF THE PPQ}

The judgemental validity (face, content and consensual validity) of the PPQ was established through the process of item generation.

The construct validity of PPQ was assessed by two ways which were derived from the literature (Abramson and Abramson, 1999) and from the expert opinion:

1. Behaviour problems of the child: The behaviour problems of a child were assumed to negatively correlate with scores of PPQ.

2. The academic performance of the child: This was assumed to be positively correlated with scores of PPQ. 
Correlations in the expected directions and the magnitudes were used to support construct validity of the instrument. Parenting was measured by the newly developed PPQ. The behaviour problems of the child were assessed by adolescent version (for 11-16 years) of SDQ validated in Tamil Language in Sri Lanka (Lukumar et al., 2008). The academic performance was obtained from the average marks of the student in the previous term exam.

Data analysis: The data analysis was done using SPSS version 15.0. Item selection procedures were applied to assess the internal consistency of the PPQ. Internal consistency was assessed by means of Cronbach's Alpha, Item-Total Correlation and Inter-Item Correlation. An alpha value between 0.7 and 0.9 was considered as evidence to support good internal consistency of the instrument (Streiner \& Norman, 2003). The mean inter item correlation above 0.3 was considered as good homogeneity among the items of scales. Both sets of data obtained on both occasions ( 2 weeks apart), were compared using Pearson's Correlation with $r>0.3$ to ensure test - retest reliability.

Construct validity was demonstrated by the associations between the parenting patterns/ quality of parenting and the selected constructs measured by the SDQ and academic performance. Pearson's correlations $(r>0.3)$ were considered as the cut off to demonstrate validity.

\section{RESULTS}

The process of development of the new instrument was described in detail under section 'Methodology'.

The internal consistency and the mean inter item correlations are shown in Table 2.
The test - retest reliability also was satisfactory (Table 3).

In the factor analysis, according to Field (2005), Kaiser recommends a KMO statistic of 0.5 as acceptable, between $0.5-0.7$ as a mediocre value, between $0.7-0.8$ as good, between $0.8-0.9$ as greater and $>0.9$ as superb. A KMO statistic of 0.673 was obtained for the present analysis which confirmed the adequacy of the sample. The Bartlett's Test of Sphericity was significant (approximate chi square $=2190.042, \mathrm{df}=$ $435, \mathrm{p}<0.000)$.

In the Principal Component Analysis (factor analysis), nine factors were extracted based on the following criteria;

1. The root criterion of selecting factors with eigenvalues greater than 1 .

2. The Scree test which is used to examine a plot of eigenvalues. The factors with eigenvalues in the sharp decent part of the plot are retained.

Based on the above criteria, 9 factors were extracted which accounted for $80.05 \%$ of the variance in the data set. These factors were rotated using Varimax rotation with Kaiser normalisation. A factor loading of 0.3 was accepted as a salient loading according to the guidelines given by Kline (1994).

Out of the nine factors, 25 out of 31 items which belonged to 3 sub-scales designed originally, loaded under relevant subscales. The other 6 items which loaded under various factors were removed after expert opinion on the importance of the items.

Table 2: Measures of internal consistency of the PPQ

\begin{tabular}{lrrr}
\hline Measure & No. of items & Cronbach's alpha Value & Mean inter item correlation \\
\hline Subscale 1 & 13 & 0.801 & 0.211 \\
Subscale 2 & 06 & 0.800 & 0.381 \\
Subscale 3 & 06 & 0.845 & 0.477 \\
Whole scale & 25 & 0.805 & 0.139 \\
\hline
\end{tabular}

Table 3: Test - retest reliability of PPQ and the subscales

\begin{tabular}{lc}
\hline Scales & Correlation Coefficient \\
\hline Subscale 1 & 0.88 \\
Subscale 2 & 0.84 \\
Subscale 3 & 0.92 \\
Whole scale & 0.87 \\
\hline
\end{tabular}


Validity: Three forms of judgemental validity (face validity, content validity and consensual validity of the PPQ) were established by the expert panel. In addition, a qualitative validation (construct validity) carried out further confirmed the validity.

The construct validity of the PPQ was assessed using the following hypothetical relationships:

1. Quality of parenting patterns was assumed to be negatively associated with behavioural problems in children as assessed by SDQ (self version for adolescents) in Tamil.

2. Quality of parenting patterns was assumed to be positively associated with the academic performance of the child assessed by average marks in the previous term exam.

The association between quality of parenting and both the hypothetical constructs was tested using Pearson's correlation coefficient (r). The cut-off level for the expected magnitude of correlation was set at \pm 0.3 , indicating a moderate correlation between the variables. The associations between the parenting patterns/ quality of parenting and the selected constructs are shown in Table 4.

Table 4: Association between measures of parenting and the hypothetical constructs (behaviour problems and academic performance of children)

\begin{tabular}{lr}
\hline Scales & Correlation Coefficient \\
\hline Subscale 1 & 0.88 \\
Subscale 2 & 0.84 \\
Subscale 3 & 0.92 \\
Whole scale & 0.87 \\
\hline
\end{tabular}

According to the results in Table 4, total difficulties measured by SDQ demonstrated a moderate correlation ( $\mathrm{r}$ $>0.3$ ) with all the scales except subscale 1 . The correlation was negative as hypothesised. The pro-social behaviour scores demonstrated a positive moderate correlation $(\mathrm{r}>$ 0.3 ), as hypothesised with all the scales except subscale 2. Academic performance by the previous term exam marks demonstrated a positive moderate correlation $(r>0.3)$, as hypothesised with all the scales except subscale 2 . The overall PPQ has demonstrated a moderate correlation ( $\mathrm{r}$ $>0.3$ ) with all the hypothetical constructs proving that PPQ is valid to measure parenting.

\section{DISCUSSION}

The tools available from reviewed literature were examined for any possibility of using those after adapting. Some tools were found to describe only a few aspects of parenting which were not comprehensive enough to describe the patterns of parenting as a whole (Larios et al., 2009; Campis et al., 1986). Even though some tools were comprehensive, they were not suitable for the local culture, as the wording and behaviours asked were not reflecting the Eastern, especially Sri Lankan Tamil culture.

Therefore, it was decided to develop a new tool and validate it. Development of the questionnaire was mainly done by devising the items from the existing tools in literature and generation of new items from the FGDs and KIIs. The same procedure was followed by researchers in Western countries in development and validation of parenting scales (Arnold et al., 1993; Campis et al., 1996; Larios et al., 2009). Sri Lankan researchers also followed the same procedures in development of tools for different purposes (Fernandopulle, 2000; Amarasinghe, 2010).

In devising the items, the wordings and events were changed with expert consultations in a way to sound familiar in the local culture. Researchers felt keeping new items generated from the FGDs and KIIs have to be given priority, as those are the reflections from the local community.

The FGDs with parents revealed that due to foreign exchange received from the relatives who visit Jaffna after the war period, the adolescents' taste has changed. According to the parents and the key informants, the priority towards education is diverted towards the things for entertainment like hand phones, computers, motorbikes etc. They further mentioned that while some children are able to get foreign money from their relatives, some have no access as such. The children who have no access for these things tend to resort to wrong ways to get those items. The parents and experts feel that the sudden entry of is sophisticated life style after a restricted life during the war period also was a reason for the adolescent issues. Even though the fear of parents that the adolescents may join the militant groups (Lukumar et al., 2008) is not there at present, still some parents are not daring enough to control the children. In a KII, a school principal said that some parents secretly ask the principal and teachers to control their children as they are afraid to control because of the fear that the children may leave the family. Though the parents want a well behaved child, their priority is not to lose the child. Parents and key informants stressed the need of interventions to parents to educate to set limits while proving their affection towards the children and to respect the children's autonomy. At the same time they prefer education to the children to make them understand the importance and affection of a parent. Most of the 
adolescents appreciate their parents and their love towards them, in the FGDs with adolescents. Most of the adolescents accept that the physical punishments are practised by the parents to discipline them. But they were not willing to accept verbal abuse, in comparison with others and punishments in front of others.

Considering all these facts, the items in the PPQ were developed. After the development of preliminary draft of the PPQ, item selection procedures were applied to remove the irrelevant items in each subscale. After applying the item selection procedures several times, a set of items which were relevant to PPQ was finalised with expert opinion.

In literature, psychometric properties of newly developed instruments were assessed by the researchers by test - retest reliability and internal consistency (Arnold et al., 1993; Campis et al., 1996; Fernandopulle, 2000; Amarasinghe, 2010).

The reliability of the PPQ was confirmed by assessing the internal consistency, the test-retest reliability and by factor analysis of the instrument. All the subscales were found to have alpha values above 0.8 , signifying high internal consistency. The overall PPQ also has an alpha value of 0.805 . These results confirmed that PPQ has a satisfactory internal consistency. The mean inter item correlation of the sub scales 2 and 3 were above 0.3 showing that homogeneity exists among the items in those subscales. The subscale 1 with mean inter item correlation of 0.211 indicates a mild correlation between the items. The mean inter item correlation of PPQ was low because the different subscales are measuring different aspects of parenting.

Even though criterion validity is considered as the gold standard, there are some measures like those related to psychology, where the criterion validity cannot be measured due to lack of a gold standard (Streiner and Norman, 2003). In such a case, construct validity would be the maximum validity achieved by various ways such as assessment of internal consistency, factor analysis, establishing convergent and discriminant validity etc (Streiner and Norman, 2003). In validating parenting scales the researchers have achieved the validity by various ways, such as achieving judgemental validity and assessing the internal consistency (Arnold et al., 1993; Campis et al., 1986; Larios et al., 2009; Sanders, 2005). In addition, Campis (1986) established construct validity to 'Parental Locus of Control Scale' against 7 scales and Arnold et al. (2003) established the construct validity against 2 measures including Child Behaviour Check List (CBCL).
As there is no gold standard to measure parenting in Eastern culture, the judgemental validity and construct validity were used to ensure the validity of PPQ. At first, the face validity of the PPQ was ensured by the expert panel, which consisted of psychiatrists, psychologists, educationists, community physicians, sociologists and religious leaders. The content validity to cover the dimensions ('connection', 'control' and 'respect') was assessed next, by the expert panel. The consensual validity was achieved by a consensus generation process using the Delphi technique with the expert panel. The construct validity of the PPQ was assessed using the following hypothetical relationships (Table 4):

1. Quality of parenting patterns was assumed to be negatively associated with behavioural problems in children as assessed by SDQ (self-administered version for adolescents) in Tamil.

2. Quality of parenting patterns is assumed to be positively associated with the academic performance of the child assessed by average marks in the previous term exam.

\section{CONCLUSIONS}

In summary, the newly developed tool, called Parenting Patterns Questionnaire (PPQ), can be considered as a valid and reliable tool to be administered in Tamil to measure the parenting patterns and the parenting quality in the Northern Province of Sri Lanka.

\section{REFERENCES}

Abramson, J. H. \& Abramson, Z. H. (1999) Survey methods in Community Medicine. 5th Ed. Edinburgh: Churchill Livingstone.

Achenbach, T. M. \& Edelbrock, C. S. (1983) Manual for the child behavior checklist and revised behavior profile. Burlington: University of Vermont Department of Psychiatry.

Amarasinghe, P. M. T. U. (2010) The assessment of life skills among the 13-15 year old, school going adolescents in Kalutara District and an interventional study to assess the effectiveness of a life skills promotion package. Colombo: Post Graduate Institute of Medicine, University of Colombo.

American Heritage Dictionary of the English language (2009) $4^{\text {th }}$ Ed. [Online] Available from: http://www.yourdictionary. com/parenting [Accessed: $06^{\text {th }}$ December 2014]

Arnold, D. S., O'Leary, S. G., Wolff, L. S., Acker, M. M. (1993) The Parenting Scale: a measure of dysfunctional parenting in 
discipline situations. Psychological Assessment, 5 (2), pp: 137144.

DOI: http://dx.doi.org/10.1037/1040-3590.5.2.137

Baumrind, D. (1967) Child care practices anteceding three patterns of preschool behavior. Genetic Psychology Monographs, 75(1), pp: 43-88.

Buri, J. R. (1991) Parental authority questionnaire, Journal of Personality Assessment, 57(1), pp: 110-119.

DOI: http://dx.doi.org/10.1207/s15327752jpa5701_13

Campis, L. K., Lyman, R. D., Prentice-Dunn, S. (1986) The parental locus of control scale: development and validation. Journal of Clinical Child Psychology, 15 (3), pp: 260 -267.

DOI: http://dx.doi.org/10.1207/s15374424jccp1503_10

Fernandopulle, P. C. (2000) Study on prevalence and risk factors for emotional abuse among school children aged 1315 years, Post Graduate Institute of Medicine, University of Colombo.

Field, A. (2005) Discovering Statistics using SPSS, 3rd Ed. London: Sage publications.

Hoffmann, J. P., Bahr, S. J. (2014) Parenting style, peer alcohol use, religiosity and adolescent heavy drinking, Journal of Studies on Alcohol and Drugs, 75 (2), pp: 222- 227.

DOI: http://dx.doi.org/10.15288/jsad.2014.75.222

Kendall, P. R. W. (1989) The Sri Lankan Tamil Community in Toronto. Toronto: City of Toronto Department of Public Health, Health Promotion and Advocacy Section.

Kline, P. (1994) An easy guide to factor analysis. London: Routledge.

Larios, S. E., Ayala, G. X., Arredondo, E. M., Baquero, B. \& Elder, J. P. (2009) Development and validation of a scale to measure Latino parenting strategies related to children's obesigenic behaviors. The parenting strategies for eating and activity scale (PEAS), Appetite, 52 (1), pp: 166- 172.

DOI: http://dx.doi.org/10.1016/j.appet.2008.09.011

Locke, H. J. \& Wallace, K. M. (1959) Short marital adjustment and prediction tests: their reliability and validity. Marriage and Family Living, 21, pp: 251-255.

DOI: http://dx.doi.org/10.2307/348022

London Safeguarding Children Board (2010) London Child protection procedures. $4^{\text {th }} \mathrm{Ed}$. [Online] Available from: http:// www.scie.org.uk/publications/adultsafeguardinglondon/files/ londonchildprotectionprocedures.pdf?res $=$ true $\left[\right.$ Accessed: $07^{\text {th }}$ October 2016]

Lukumar, P., Wijewardana, K., Hermansson, J. \& Lindmark, G. (2008) Validity and reliability of Tamil version of strengths and difficulties questionnaire self report, Ceylon Medical Journal, 53(2), pp: 48-52.

DOI: http://dx.doi.org/10.4038/cmj.v53i2.232

Maccoby, E. E., Martin, J. A. (1983) Socialization in the context of family: parent-child interaction. Hand book of Child Psychology. Volume 4, New York: Wiley.

Russell, S. T., Crockett, L. J. \& Chao, R. (Eds.) (2010) Asian American parenting and parent-adolescent relationships. New York: Springer.

Sanders, M. (2005) Parent behavior questionnaire and the parent behavior frequency questionnaire: psychometric characteristics. Dissertation. New York: Pace University.

Santrock, J. W. (2014) A topical approach to life-span development, $7^{\text {th }}$ Ed. New York: McGraw-Hill.

Sivarajah, R. (1998) From family to state: six tamil women receiving social assistance, Master's Thesis, North York, Ontario: York University.

Stafström, M. (2014) Influence of parental alcohol related attitudes, behaviour and parenting styles on alcohol use in late and very late adolescence. European addiction Research, 20(5), pp: 233-240.

DOI: http://dx.doi.org/10.1159/000357319

Strayhorn, J. M., Weidman, C. S. (1988) A parent practices scale. Journal of the American Academy of Child and Adolescent Psychiatry, 27(5), pp: 613-618.

DOI: http://dx.doi.org/10.1097/00004583-198809000-00016

Streiner, D. L. \& Norman G. R. (2003) Health measurement scales: a practical guide to their development and use. $4^{\text {th }} \mathrm{Ed}$. Oxford: Oxford University Press.

Tyyskä, V. \& Colavecchia, S. (2001) Report on individual interviews in Toronto: study of parenting issues of newcomer families in Ontario, Report for the Centre for Research and Education in Human Services and the Joint Centre for Excellence for Research on Immigration and Settlement. Kitchener-Waterloo: Centre for Research and Education in Human Services.

White, A. (2005) Assessment of parenting capacity: literature review, Centre for Parenting and Research-NSW department of community services. [Online] Available from: http://www. community.nsw.gov.au/_data/assets/pdf_file/0020/321635/ research_parenting_capacity.pdf [Accessed: 10 $10^{\text {th }}$ February 2015]

WHO (2007) Helping parents in developing countries improve adolescents' health. [Online] Available from: http://apps.who.int/iris/ bitstream/10665/43725/1/9789241595841_eng. pdf?ua $=1 \& u a=1$ [Accessed: $08^{\text {th }}$ October 2016] 